\title{
Combining Resting-state fMRI and DTI Analysis for Early-onset Schizophrenia
}

\author{
Ming Ke ${ }^{1,2}$, Hui Shen ${ }^{2,3}$, Jintu Fan ${ }^{3}$, Xing Huang ${ }^{2}$, Zongtan Zhou ${ }^{2}$, Xiaogang Chen 4 * Dewen Hu ${ }^{2 \dagger}$ \\ ${ }^{1}$ College of Computer and Communication, Lanzhou University of Technology, \\ Lanzhou, Gansu, 730050, P.R.China \\ ${ }^{2}$ College of Mechatronics and Automation, National University of Defense Technology, \\ Changsha, Hunan, 410073, P.R.China \\ ${ }^{3}$ Institute of Textiles and Clothing, The Hong Kong Polytechnic University, \\ Hung Hom, Kowloon, Hong Kong \\ ${ }^{4}$ Institute of Mental Health, The Second Xiangya Hospital, Central South University, \\ Changsha, Hunan, 410073, P.R.China \\ Received: $27 / 12 / 08$ \\ Accepted: 18/09/09
}

\begin{abstract}
We combined measures of resting-state functional magnetic resonance imaging and diffusion tensor imaging (DTI) to investigate alterations of function-structure relationships in patients with early-onset schizophrenia. DTI analysis revealed reduced fractional anisotropy in right frontal white matter. Corresponding gray matter regions showed reduced functional connectivity with other regions in the brain in patients with early-onset schizophrenia, compared with healthy controls. Our results demonstrated abnormal function-structure relationships in early-onset schizophrenia, and supported the view that white matter lesions might disrupt the neural circuits between frontal regions and other brain regions, and affect the functional connectivity in the frontal cortex.
\end{abstract}

Keywords: Functional connectivity, Anatomical connectivity, Resting fMRI, Fractional anisotropy, Earlyonset schizophrenia.

\section{Introduction}

The exploration of the relationship between neural function and structure is central for research in both healthy subjects and patient populations, such as schizophrenia. Advances in neuroimaging technology allow for the collection of both structural and functional data from human subjects (Ref. 1) and provide information on brain abnormalities in schizophrenia research (Ref. 2).

Functional magnetic resonance imaging (fMRI) has become one of the most widely adopted methods using blood oxygenation level dependent (BOLD) signal contrast, because of its non-invasive and high spatial resolution (Ref. 3). fMRI is widely used to detect brain regions that change their level of activation in response to tasks, but can also measure correlations in spontaneous fluctuations of signals between different brain regions, and reveal functionally connected circuits (Ref. 4). More recently, the functional activity and connectivity of the brain during the resting state have attracted increasing

*Corresponding author, E-mail: chenxghu@yahoo.com.cn (X. Chen).

${ }^{\dagger}$ Corresponding author, E-mail: dwhu@nudt.edu.cn (D. Hu). 
attention. Resting-state fMRI studies have identified associated fluctuations in brain regions involved in motor, auditory, visual, and language function (Ref. 5, 6, 7, 8, 9). Resting-state neuroimaging measures were utilized to investigate the relationship between patterns of functional connectivity and schizophrenia. Some investigators have reported that the activity of distributed regions is spontaneously increased or decreased in functionalanatomic networks during the resting state. (Ref. 2, 10). This suggests that resting activity patterns can reflect functional activity that is as important as the activity evoked by tasks (Ref. 11). Aberrant connectivity distributed widely throughout the entire brain in patients with schizophrenia have been reported (Ref. 12). Furthermore, self-generated mental activity at rest is thought to be potentially relevant to the underlying neuropathological mechanisms of schizophrenia (Ref. 10).

Diffusion tensor imaging (DTI) is a non-invasive technique derived from diffusion magnetic resonance imaging (Ref. 13). It can provide unique biologically and clinically relevant information. This information includes parameters that help characterize tissue composition, the physical properties of tissue constituents, tissue microstructure and its architectural organization (i.e. the myelinated fiber tracts) by measuring the directionality and distribution of water molecules across tissue components (Ref. 14, 15). Signal intensities in DTI sensitized to the diffusion of water molecules along a single diffusionencoding direction (Ref. 13). Recently, DTI has been increasingly applied to investigating pathways of anatomical connectivity in vivo (Ref. 15, 16). By quantifying measures of tissue water diffusion anisotropy, DTI can reflect the bulk integrity and orientation of neural tissue at the imaging voxel level (Ref. 15). To describe the anisotropy of diffusion, Basser et al. proposed the commonly used indices including relative anisotropy (RA), fractional anisotropy (FA), and volume ratio (VR) (Ref. 17). FA is a measure of the fraction of the magnitude of the tensor that can be ascribed to anisotropic diffusion. It is deviation from pure isotropic diffusion of water mobility in vivo. FA provides information about the myelination, density, coherence and integrity of fibers. FA values are thought to reflect microstructural obstacles including the integrity of axonal cell membranes, the amount and integrity of myelin around the axons (Ref. 1). There are growing evidences that the pathophysiology of schizophrenia reflects a disturbance in the white matter and FA tends to decrease suggests a corresponding decline in the axonal conduction and the efficiency of information transfer distributed cortical networks (Ref. 18, 19, 20). Alterations in diffusion and anisotropy of white matter (WM) can result from various disease processes or abnormal development (Ref. 21). Thus, the development of DTI provides an appropriate tool for investigating WM abnormalities in schizophrenia (Ref. 16, 22).

A small number of studies have combined measures of task-related fMRI and DTI to investigate function-structure relationships in healthy subjects and patients with neurological diseases and psychological disorders (Ref. 1, 19, 23). Toosy et al. reported that the mean FA in the optic radiations found using a tractography algorithm was correlated with an fMRI measure of visual cortex activity (Ref. 23). Another study by Baird et al. explored reaction time in relation to cortical activity and the integrity of the fibers (Ref. 1). Some studies have also suggested a correlation between the WM abnormalities and brain activation in schizophrenia during working memory tasks (Ref. 19). These investigations have demonstrated that combining fMRI and DTI is an effective methodology for investigating function and structure in human brain.

Schizophrenia is a severe mental disorder that affects approximately $1 \%$ of the general population, with often devastating effects on the psychological wellbeing and financial resource of the patients, families, and the larger community (Ref. 2). Hallucinations and delusions are hallmark features in psychotic disorder of schizophrenia, and the disease is characterized by a range of symptoms. Earlyonset schizophrenia is defined as schizophrenia with an onset of psychosis before the age of 18 years, and displays many of the same neurobiological abnormalities observed in adult-onset schizophrenia (Ref. 24). A review by Nicolson argued that earlyonset schizophrenia was clinically and biologically 
continuous with adult-onset schizophrenia (Ref. 25). However, some studies have reported that patients with early-onset schizophrenia tend to exhibit a more severe form of the disorder than adult patients (Ref. 24, 26). Currently, the underlying pathology of early-onset schizophrenia remains largely unknown.

In this study, we combined resting-state fMRI and DTI to investigate changes in WM structure in relation to altered patterns of functional connectivity in gray matter (GM) during the resting state. We applied DTI to assess anisotropy changes of WM in the brains of patients with early-onset schizophrenia. Then, with the aim of identifying functional networks that are dependent on the affected structures, we analyzed functional connectivity between GM corresponding to the abnormal WM and the rest of the GM in the brain. Finally, we discuss potential relationships between function and structure in early-onset schizophrenia.

\section{Methods and materials}

\subsection{Subjects}

Twenty-four patients diagnosed with early-onset schizophrenia (nine girls, 15 boys; ages 14-18 years, mean age 15.80 years, S.D. 1.10 years) participated in this study, which satisfied the Diagnostic and Statistical Manual of Mental Disorders, Fourth Edition (DSM-IV; American Psychiatric Association, 1994) criteria for schizophrenia. All patients were recruited from outpatient departments and inpatient units at the department of Psychiatry, Second Xiangya Hospital of Central South University of China, between October 2006 and March 2007.

Thirty-one healthy participants were recruited from advertisements and community centers (18 girls, 13 boys; ages $14-18$ years, mean age 16.26 years, S.D. 1.00 years), and matched to the patients on age, gender and education. All of the participants were right-handed. None of them had experienced major head trauma, had a history of alcohol/drug dependence, or a history of neurological disorder.

Written informed consent was obtained from all participants and their parents. This study was approved by the Medical Research Ethics Committee of the Second Xiangya Hospital, Central South University.

\subsection{Data acquisition}

Functional magnetic resonance imaging and diffusion tensor imaging scans were acquired on a 1.5T GE Signa System (GE Signa, Milwaukee, Wisconsin, USA) at Second Xiangya Hospital of Central South University.

Participants were instructed to lie quietly with eyes closed while remaining awake during the experiment, and to perform no specific cognitive exercise. After the session, participants were asked whether they were awake in the previous session. All participants confirmed they were awake.

To reduce head motion, participants' heads were fixed using foam pads, with a standard birdcage head coil. The structural images were acquired axially with the following parameters: repetition time (TR) $=1924 \mathrm{~ms}$, echo time (TE) $=7.5 \mathrm{~ms}$, field of view $(F O V)=24 \mathrm{~cm}$, flip angle $(\mathrm{FA})=90^{\circ}$, matrix $=256 \times 256$, slice thickness $=5 \mathrm{~mm}$, gap $=1 \mathrm{~mm}$. Functional MRI images were collected in the same location as the structural slices, using a gradient-echo echo-planar imaging (EPI) sequence: $\mathrm{TR}=2000 \mathrm{~ms}$, TE=40 ms, $\mathrm{FOV}=24 \mathrm{~cm}, \mathrm{FA}=90^{\circ}$, matrix $=64 \times 64$, slice thickness $=5 \mathrm{~mm}$, gap $=1 \mathrm{~mm}$, a volume $=20$ axial levels. Each functional restingstate session lasted six minutes, and 180 volumes were obtained.

Diffusion tensor images were acquired by a single-shot EPI sequence with the following parameters: $\mathrm{TR}=12000 \mathrm{~ms}, \mathrm{TE}=105 \mathrm{~ms}, \mathrm{FOV}=24 \mathrm{~cm}$, $\mathrm{NEX}=5, b=1000 \mathrm{~s} / \mathrm{mm}^{2}, 30$ contiguous axial slices. Diffusion was measured in thirteen directions, plus one image with no diffusion weighting. The acquired image matrix was $128 \times 128 \times 30$ and was subsequently interpolated to $256 \times 256 \times 30$, giving a reconstructed voxel resolution of $0.94 \times 0.94 \times 4 \mathrm{~mm}^{3}$.

\subsection{Data analysis}

The procedures in this study included two steps: (i) Diffusion tensor image analysis aimed at identifying the areas exhibiting abnormal brain white matter; (ii) Functional connectivity analysis was for de- 


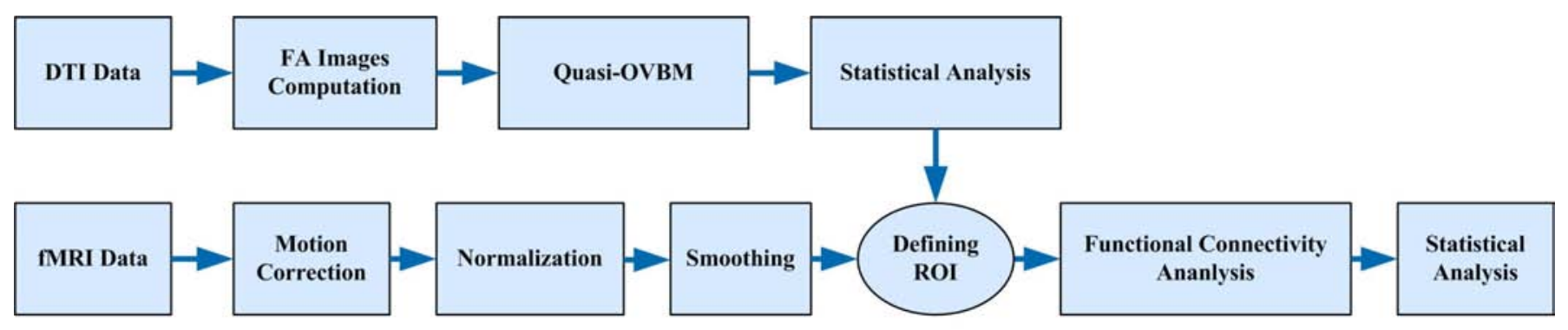

Figure 1: Data analysis procedures for combined fMRI data and DTI data.

tecting areas showing differences of functional connectivity. A diagram of the method was shown in Figure 1.

\subsubsection{Diffusion tensor image analysis}

Before tensor calculation, the raw DTI data sets were first realigned to minimize misregistration due to participant head motion. The diffusion tensor matrix was then calculated according to the Stejskal and Tanner equation (Ref. 27, 28).

The Stejskal-Tanner imaging sequences were measured by the echo magnitudes of the diffusion weighted signals in the $M$ direction $\left(S_{M}\right)$, and the echo magnitudes of the non-diffusion weighted signals $\left(S_{0}\right)$.

$$
S_{M}=S_{0} e^{-b D}
$$

where $b$ is diffusion weighted factor and $D$ is the water diffusion constant.

The constant $b$ was defined by:

$$
b=\gamma^{2} \delta^{2} G^{2}(\Delta-\delta / 3)
$$

where $\gamma$ is the gyromagnetic ratio, $\delta$ and $G$ is the duration and signal intensity of the diffusion sensitizing gradient pulse along a given direction, $\Delta$ represents the time interval between the diffusion gradient pulses.

For anisotropic samples, D was a $3 \times 3$ diffusion tensor,

$$
D=\left[\begin{array}{lll}
D_{x x} & D_{x y} & D_{x z} \\
D_{y x} & D_{y y} & D_{y z} \\
D_{z x} & D_{z y} & D_{z z}
\end{array}\right]
$$

The diffusion tensor is symmetric and thus has six independent variables.

Equation (1) had to be rewritten in a more general form:

$$
S_{M}=S_{0} e^{-\sum_{i} \sum_{j} b g_{i j} D_{i j}}
$$

where $g$ is the normalized diffusion sensitizing gradient vector, i.e. $g=\left(g_{x}, g_{y}, g_{z}\right)^{T}=G /|G|$.

The apparent diffusion coefficient (ADC) in every direction was computed by performing a logarithmic transformation:

$$
A D C_{M}=-\frac{1}{b} \ln \left(\frac{S_{M}}{S_{0}}\right)
$$

Next, the symmetric diffusion tensor $D$ was estimated using multivariate linear regression of equation (6) (Ref. 29):

$$
\left[\begin{array}{c}
A D C_{1} \\
A D C_{2} \\
\vdots \\
A D C_{M}
\end{array}\right]=G *\left[\begin{array}{c}
D_{x x} \\
D_{x y} \\
D_{x z} \\
D_{y y} \\
D_{y z} \\
D_{z z}
\end{array}\right]
$$

where $D_{x y}=D_{y x}, D_{x z}=D_{z x}, D_{y z}=D_{z y}$.

From the diffusion tensor, three eigenvectors $\varepsilon_{1}$, $\varepsilon_{2}, \varepsilon_{3}$ and the corresponding three eigenvalues $\lambda_{1}$, $\lambda_{2}, \lambda_{3}$ were obtain by diagonalizing the tensor for each voxel, i.e., $|\lambda I-D|=0$, where $I$ represents third-order unit matrix. These three eigenvalues represent the magnitude of diffusivity in the three directions. Finally, FA values were computed in a voxelbased fashion using equation (7) and FA maps for each subject were generated: 


$$
F A=\sqrt{\frac{3\left[\left(\lambda_{1}-\bar{\lambda}\right)^{2}+\left(\lambda_{2}-\bar{\lambda}\right)^{2}+\left(\lambda_{3}-\bar{\lambda}\right)^{2}\right]}{2\left(\lambda_{1}^{2}+\lambda_{2}^{2}+\lambda_{3}^{2}\right)}}
$$

where $\bar{\lambda}=\left(\lambda_{1}+\lambda_{2}+\lambda_{3}\right) / 3$.

We propose a method called quasi-OVBM (Ref. 30) to normalize FA images on the basis of optimized voxel-based morphometry (OVBM) (Ref. 31), where the raw non-diffusion-weighted image ( $b 0$ image) of each subject was used as a T1 structural image. The procedure contained two steps (Fig. 2).

One step was to create the user-template as follows: (a) The raw $b 0$ images of the subjects were segmented into GM, WM, and cerebrospinal fluid. (b) The WM images were normalized using the standard WM template. The transformation matrices were then applied to normalization of the raw $b 0$ images. (c) The normalized $b 0$ images were segmented and the segmented images were smoothed with an $8 \mathrm{~mm}$ full-width half-maximum (FWHM) Gaussian kernel. This process served to create the user-template.

The second step was to normalize the FA images using 12-parameter affine transformation. The optimal 12-parameter affine transformation was obtained by normalizing each WM image to the user WM template, which resulted from the repeated segmentation of the raw $b 0$ images. In addition, each FA image was smoothed with a Gaussian kernel of $8 \mathrm{~mm}$ FWHM.

In statistical analysis, a two-sample $t$-test framework was used to contrast the FA maps. Statistical significance was evaluated between patients with early-onset schizophrenia and healthy controls. FA reductions were tested in patients across the entire volume $(P<0.005$, uncorrected).

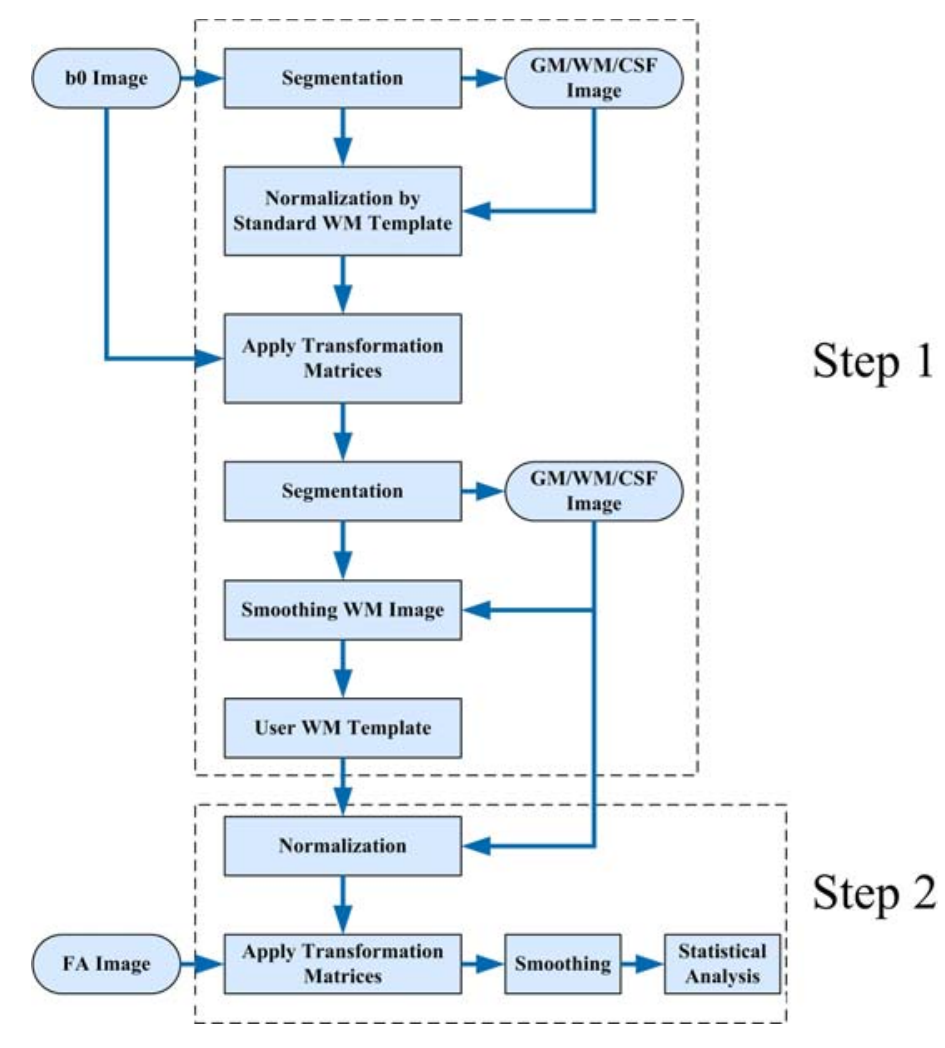

Fig. 2. Data processing flowchart for quasi-OVBM.

\subsubsection{Functional connectivity analysis}

The corresponding functional MRI data sets were preprocessed using the statistical parametric mapping software package (SPM2, Wellcome Department of Cognitive Neurology, London, UK). For head motion correction, the echo planar imaging volumes were realigned to the first image by registering and reslicing and a mean functional image was correspondingly obtained. All the subjects in this study had less than $1 \mathrm{~mm}$ translation in $x, y$, or $z$ axis and $1^{\circ}$ of rotation in each axis. None of the analyzed subjects showed gross distorting head movement. The mean image was registered to the MNI152 (Montreal Neurological Institute) standard space using affine registration. Then all functional volumes were normalized by affine transformation and the data were resampled at $2 \times 2 \times 2 \mathrm{~mm}^{3}$. Finally, spatial filtering with an $8 \mathrm{~mm}$ Gaussian ker- 
nel and temporal filtering with a band-pass filter $(0.01<f<0.08 \mathrm{~Hz})$ (Ref. 5) were performed for decrease spatial noise and high-frequency noise.

In early-onset schizophrenic patients, two clusters exhibiting FA reductions were found in the medial frontal gyrus and subgyral area of the right frontal region as measured by DTI. We respectively defined these two regions of GM as the regions of interest (ROI) using software developed in the Functional MRI Laboratory at the Wake Forest University School of Medicine (Ref. 32). We applied intersection of two standard template of the Automated Anatomical Labeling atlas (Ref. 33)and the Talairach Daemon atlas to create these two ROIs.

Functional connectivity analysis was performed for these two GM regions. The reference time course for each region of interest was obtained by averaging the time courses of all voxels within the region of interest. Correlations between the reference GM regions and the rest of the brain in a voxelbased manner were then computed using Pearson's correlation coefficients method:

$$
c c=\frac{\sum(r-\bar{r})(R-\bar{R})}{\sqrt{\sum(r-\bar{r})^{2}} \sqrt{\sum(R-\bar{R})^{2}}}
$$

where $R$ denotes the reference time course, and $r$ denotes one of the time courses of the rest of the voxels in the brain. $\bar{R}$ and $\bar{r}$ refer to the time averages of $R$ and $r$ respectively. These correlation coefficients were then converted to $z$ values using Fisher's $z$-transform to improve the normality of correlation coefficients:

$$
z=\left(\frac{1}{2}\right) \log _{e}\left[\frac{1+c c}{1-c c}\right]
$$

Differences between the schizophrenic and healthy groups were examined using two-sample $t$ tests at each voxel on the corrected $z$ values. Voxels that had p-values of less than 0.001 (uncorrected) and a spatially contiguous cluster size of 20 voxels or greater were considered to be significantly different.

\section{Results}

\subsection{Comparison of mean fractional anisotropy between patients and controls}

When the mean fractional anisotropy between the two groups was compared, the patients showed significantly lower FA values in the right frontal lobe compared with the controls. Figure 3 shows the localization of FA reduced regions, as well as plots of mean FA values for each cluster. Two clusters were included in the right medial frontal gyrus $\{$ peak coordinate (MNI) $[x=10, y=56, z=-16]$, peak $t=3.57\}$, and the subgyral region of the right frontal area \{peak coordinate (MNI) $[x=12, y=44, z=-16]$, peak $t=3.44\}$. In Figure 3b, mean FA values of these two right frontal areas in the controls were significantly higher than in the patients $(0.26$ and 0.26 in controls and 0.21 and 0.19 in patients).

\subsection{Comparison of functional connectivity between patients and controls}

By comparing two seed regions and their associated correlation maps between two groups, we found significantly decreased functional connectivity in patients compared with healthy controls. In the functional connectivity maps of the right medial frontal gyrus, the right precentral gyrus, left inferior occipital gyrus and bilateral cerebellum revealed a significantly reduced functional connectivity in the schizophrenic group (Fig. 4 and Table 1). In the functional connectivity maps of right subgyral region, patients revealed significantly reduced functional connectivity in the bilateral inferior occipital gyrus and middle temporal gyrus, right superior frontal gyrus, left middle frontal gyrus, left inferior temporal gurus, caudate, left anterior cingulate and bilateral cerebellum (Fig. 5 and Table 2). These results demonstrated that several frontal cortices were decreased in their correlation with these two GM regions. In addition, the temporal lobe and occipital lobe extending to the cerebellum were also decreased in their correlation with the ROIs in patients with early-onset schizophrenia. 
(a)
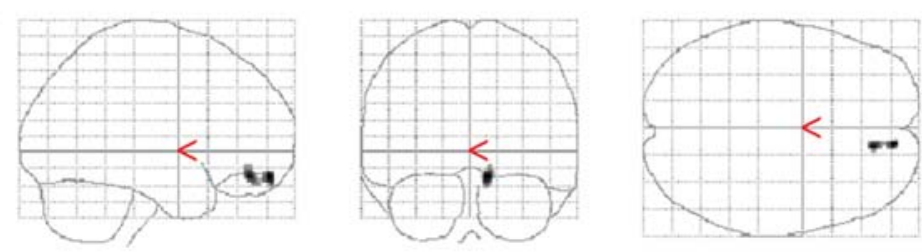

(b)
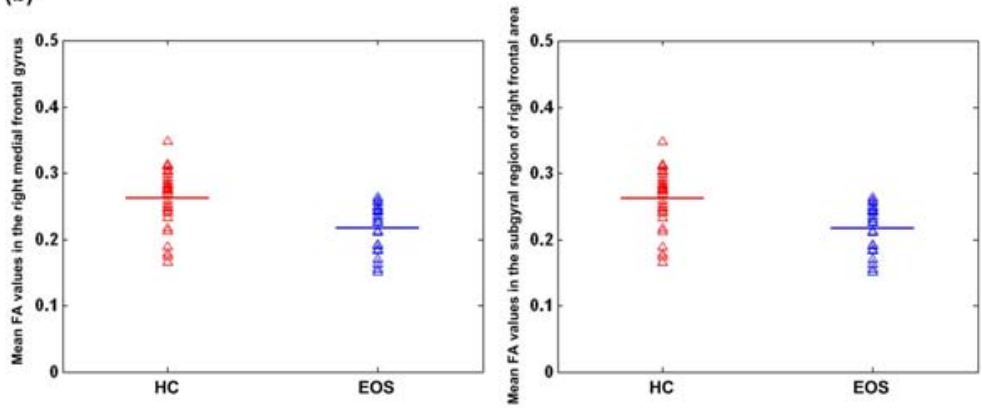

Figure 3: DTI analysis revealing significant FA differences between patients with early-onset schizophrenia and healthy controls in the right medial frontal gyrus and subgyral region of the right frontal area. (a) shows the voxels as maximum intensity projections $\left(P<0.005\right.$, uncorrected; minimum cluster size $\left.=80 \mathrm{~mm}^{3}\right)$. (b) shows mean individual FA values for healthy controls (HC) and patients with early-onset schizophrenia (EOS) in the medial frontal gyrus and subgyral region of the right frontal area, respectively.

Table 1. Brain regions showing reduced connectivity in patients with early-onset schizophrenia relative to healthy controls in right medial frontal gyrus maps

\begin{tabular}{ccccccc}
\hline & & Cluster & \multicolumn{3}{c}{ MNI coordinates } & Peak \\
\cline { 5 - 6 } Region & BA & size & $\mathrm{x}$ & $\mathrm{y}$ & $\mathrm{z}$ & $\mathrm{t}$ \\
\hline preCG & BA6(R) & 288 & 40 & -8 & 32 & 4.48 \\
IOG & BA18(L) & 424 & -36 & -88 & -16 & 3.95 \\
Cerp & $\mathrm{L}$ & 160 & -32 & -48 & -38 & 3.78 \\
Cerp & $\mathrm{R}$ & 168 & 56 & -56 & -26 & 3.91 \\
Cerp & $\mathrm{R}$ & 1224 & 12 & -74 & -48 & 3.77 \\
\hline
\end{tabular}

Note. BA, Brodmann's area; L, left hemisphere; R, right hemisphere; Cluster size in $\mathrm{mm}^{3}$. Abbreviations-preCG: precentral gyrus; IOG: Inferior occipital gyrus; Cerp: Cerebellar posterior lobe
Table 2. Brain regions showing reduced connectivity in patients with early-onset schizophrenia relative to healthy controls in right subgyral maps

\begin{tabular}{ccccccc}
\hline & & Cluster & \multicolumn{3}{c}{ MNI coordinates } & Peak \\
\cline { 5 - 6 } Region & BA & size & $\mathrm{x}$ & $\mathrm{y}$ & $\mathrm{z}$ & $\mathrm{t}$ \\
\hline IOG & BA17(R) & 1248 & 30 & -88 & -20 & 5.30 \\
IOG & BA17(L) & 312 & -38 & -94 & -8 & 4.37 \\
SFG & BA6(R) & 336 & -4 & 4 & 74 & 4.69 \\
MFG & BA6(L) & 216 & -44 & 2 & 54 & 4.05 \\
MTG & BA21(R) & 168 & 68 & -14 & -10 & 3.80 \\
MTG & BA21(L) & 192 & -64 & -16 & -6 & 3.69 \\
ITG & BA20(L) & 176 & -54 & -2 & -34 & 3.66 \\
Cau & L & 168 & -8 & 2 & 14 & 4.60 \\
ACC & BA25(L) & 228 & -6 & 20 & -4 & 4.34 \\
ACC & BA32(L) & 208 & -6 & 42 & 16 & 3.78 \\
Cerp & L & 240 & -20 & -70 & -50 & 4.27 \\
Cerp & R & 552 & 24 & -80 & -44 & 3.98 \\
\hline
\end{tabular}

Note. BA, Brodmann's area; L, left hemisphere; R, right hemisphere; Cluster size in $\mathrm{mm}^{3}$. Abbreviations-IOG:Inferior occipital gyrus; SFG:Superior frontal gyrus; MFG:Middle frontal gyrus; MTG:Middle temporal gyrus; ITG:Inferior temporal gyrus; Cau:Caudate; ACC:Anterior cingulate cortex; Cerp:Cerebellar posterior lobe 


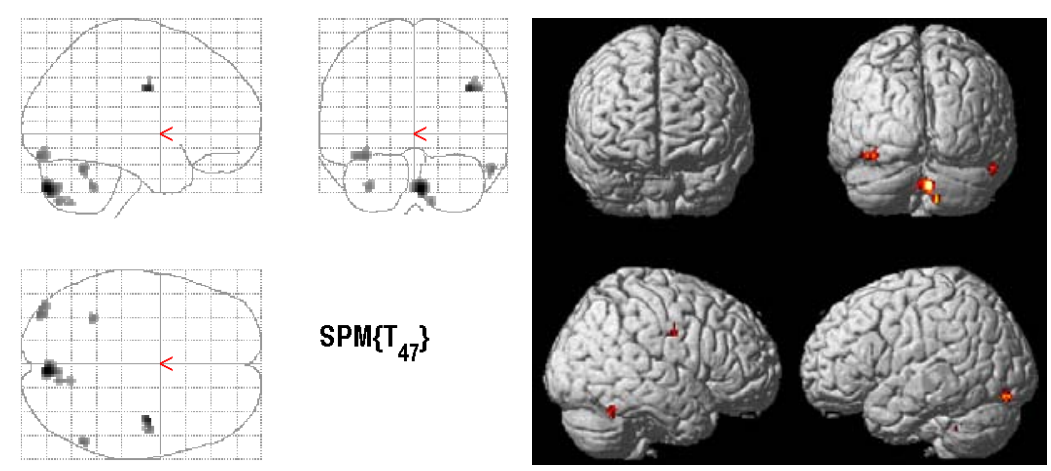

Figure 4: $\operatorname{SPM}\{t\}$ of right medial frontal gyrus maps showing statistically significant differences in functional connectivity between patients with early-onset schizophrenia and healthy controls during resting state. The statistical map was thresholded at $P<0.001$ (uncorrected) and cluster size of at least $160 \mathrm{~mm}^{3}$.
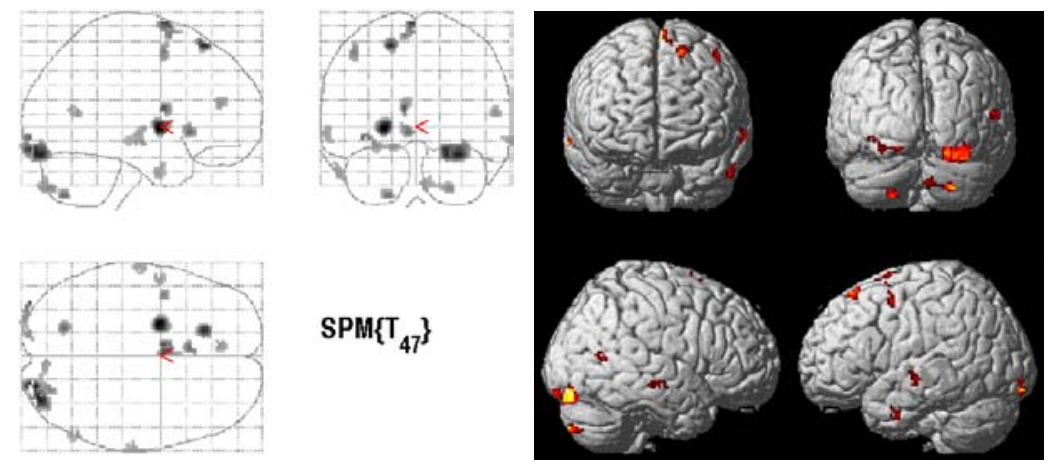

Figure 5: $\operatorname{SPM}\{t\}$ of right subgyral maps showing statistically significant differences in functional connectivity between patients with early-onset schizophrenia and healthy controls during the resting state. The statistical map was thresholded at $P<0.001$ (uncorrected) and cluster size of at least $160 \mathrm{~mm}^{3}$.

\section{Discussion}

We examined differences of the mean FA between healthy controls and patients with early-onset schizophrenia, and found that FA was significantly reduced in two WM regions of the right frontal lobe in patients. We then extracted the BOLD signals at rest in these two corresponding regions of reference in the GM. The correlation between two regions of interest and all other brain regions was then analyzed to explore differences in functional connectivity between early-onset schizophrenic patients and healthy controls. Unlike a former study combining DTI and fMRI on schizophrenia (Ref. 19), we analyzed the resting-state functional connectivity patterns of the early-onset schizophrenia from a functional integration perspective, and selected the regions of interest based on the results from DTI. To our knowledge, this is the first study examining early-onset schizophrenia using an analysis of functional connectivity with a combination of restingstate fMRI and DTI measures. Three major novel findings were generated by this study.

First, we applied a quasi-OVBM method in DTI for normalizing FA images and compared the differences in FA in the whole brain between healthy controls and patients with early-onset schizophrenia. This method avoided errors of interpretation caused by misclassification of non-brain tissue and could detect anatomically variable structures on a voxel 
by voxel basis, independent of user bias (Ref. 31). Compared with controls, patients exhibited significantly decreased FA diffusion in two brain regions of the right frontal lobe (Ref. 16, 22), including the medial frontal gyrus and the subgyral region of the right frontal lobe. In addition, we compared individual FA values in the medial frontal gyrus and the subgyral region of the right frontal area between the patients and controls. The mean FA values for two clusters in the patients group ( 0.21 and 0.19$)$ were lower than in the controls group (0.26 and 0.26). Since a reduced FA implied differences in fiber architecture or myelinization of the fibers (Ref. 19), our results indicate a disruption of WM integrity in the frontal areas of patients, and suggest that these deficits might affect anatomical connectivity in a neural network.

Second, the correlation in spontaneous slow fluctuations during the resting state between the two corresponding regions of reference in the GM and all other brain regions were compared between the patients and controls. A number of previous studies have applied resting-state fMRI to examine the functional connectivity in healthy subjects as well as in patients with various diseases (Ref. 5, $6,11)$. In addition, spontaneous low fluctuations observed in the resting state have been attributed to spontaneous neural activity (Ref. 5). In adultonset schizophrenia, dysfunction of the frontalcerebellar network, frontal-temporal network, and frontal-occipital network have been reported in previous studies (Ref. 34). Similarly, in the present study, our findings demonstrated that patients with early-onset schizophrenia showed a significantly reduced functional connectivity between spontaneous slow fluctuations of frontal lobe activity and that of other brain areas in the frontal-cerebellar, frontaltemporal, and frontal-occipital regions. Our results demonstrated that disturbance in early neural development in early-onset schizophrenia patients might lead to a predisposition for the adult disorder (Ref. 26, 35). A longitudinal study between earlyonset and adult schizophrenia are required to further investigate the features of the pathophysiology of schizophrenia.

Third, we described an idea that specified the anatomical regions defined using DTI, to inform functional connectivity in order to explore the function-structure relationships in early-onset schizophrenia. Anatomical connectivity and functional connectivity are two fundamental research questions in neuroscience. The results of this study revealed that reduced FA values in two frontal clusters were related to abnormal functional connectivity between frontal regions and other separate brain regions. Utilizing fMRI and DTI to draw population level inferences could help to reveal the relationships between structure and function (Ref. 23). Our results demonstrated that altered functional patterns were relative to specific WM changes in early-onset schizophrenia. These findings suggest that structural deficits might affect functional connectivity within neural networks and functional activity in remote areas (Ref. 19).

In summary, our analysis not only demonstrated relationships between changes in WM and alterations in functional connectivity, but also added to our understanding of the underlying mechanism of pathology in early-onset schizophrenia. Further longitudinal studies should investigate the progression of patients through the course of the disease, to enable predictions about severe cases of schizophrenia.

\section{Acknowledgments}

The authors would like to thank the anonymous reviewers for their constructive comments and suggestions, which greatly improved the paper. This work is supported by National Basic Research Program of China (2007CB311001), Natural Science Foundation of China (60771062, 90820304, 60835005), Hong Kong Polytechnic University's Niche Area project (J-BB6T), Program for New Century Excellent Talents in University (NCET-08-0147).

\section{References}

1. A.A. Baird, M.K. Colvin, J.D. VanHorn, S. Inati, M.S. Gazzaniga, "Functional connectivity: Integrating behavioral, diffusion tensor imaging, and functional magnetic resonance imaging data sets," J. Cognitive Neuroscience, 17, 687-693 (2005). 
2. M.A. Niznikiewicz, M. Kubicki, M.E. Shenton, "Recent structural and functional imaging findings in schizophrenia," Curr. Opin. Psychiatry, 16, 123-147 (2003).

3. S. Ogawa, R.S.Menon, D.W. Tank, S.G. Kim, H. Merkle, J.M. Ellerman, K. Ugurbil, "Functional brain mapping by blood oxygenation level-dependent contrast magnetic resonance imaging," Biophys. J., 64, 803-812 (1993).

4. B.P. Rogers, V.L. Morgan, A.T. Newton, J.C. Gore, "Assessing functional connectivity in the human brain by fMRI," Magn. Reson. Imaging, 17, 687-693 (2007).

5. B. Biswal, F.Z. Yetkin, V.M. Haughton, J.S. Hyde, "Functional connectivity in the motor cortex of resting human brain using echo-planar MRI," Magn. Reson. Med., 34, 537-541 (1995).

6. M.J. Lowe, M.D. Phillips, J.T. Lurito, D. Mattson, M. Dzemidzic, V.P. Mathews, "Multiple sclerosis: low-frequency temporal blood oxygen leveldependent fluctuations indicate reduced functional connectivity initial results," Radiology, 224, 184-192 (2002).

7. D. Cordes, V.M. Haughton, K. Arfanakis, G.J. Wendt, P.A. Turski, C.H.Moritz, M.A. Quigley, M.E. Meyerand, "Mapping functionally related regions of brain with functional connectivity MR imaging," Am. J. Neuroradiol., 21, 1636-1644 (2000).

8. M. Hampson, B.S. Peterson, P. Skudlarski, J.C. Gatenby, J.C. Gore, "Detection of functional connectivity using temporal correlations in MR images," Hum. Brain Mapp., 15, 247-262 (2002).

9. V.G. van de Ven, E. Formisano, D. Prvulovic, C.H. Roeder, D.E.J. Linden, "Functional connectivity as revealed by spatial independent component analysis of fMRI measurements during rest," Hum. Brain Mapp., 22, 165-178 (2004).

10. D. Malaspina, J. Harkavy-Friedman, C. Corcoran, L. Mujica-Parodi, D. Printz, J.M. Gorman, R.V. Heertum, "Resting neural activity distinguishes subgroups of schizophrenia patients," Biol. Psychiatry, 56, 931937 (2004).

11. R.L. Buckner and J.L. Vincent, "Unrest at rest: the importance of default activity and spontaneous network correlations," NeuroImage, 37, 1091-1096 (2007).

12. R.L. Bluhm, J. Miller, R.A. Lanius, E.A. Osuch, K. Boksman, R.W.J. Neufeld, J. Théberge, B. Schiaefer, P. Williamson, " Spontaneous low-frequency fluctuations in the BOLD signal in schizophrenic patients: anomalies in the default network," Schiizophr. Bull., 33, 1004-1012 (2007).

13. N. Ramnani, T.E.J. Behrens, W. Penny, P.M., Matthews, "New approaches for exploring anatomical and functional connectivity," Biol. Psychiatry, 58, 921-929 (2004).

14. P.J. Basser, D.K. Jones, "Diffusion-tensor MRI: the- ory, experimental design and data analysis-a technical review," NMR Biomed., 15, 456-467 (2002).

15. C. Pierpaoli, P. Jezzard, P.J. Basser, A. Barnett, G.D. Chiro, "Diffusion tensor MR imaging of the human brain," Radiology, 201, 637-648 (1996).

16. R.A.A. Kanaan, J. Kim, W.E. Kaufmann, G.D. Pearlson, G.J. Barker, P.K. McGuire, "Diffusion tensor imaging in schizophrenia," Biol. Psychiatry, 58, 921929 (2005).

17. P.J. Basser and C. Pierpaoli, "Microstructural and physiological features of tissues elucidated by quantitative-diffusion-tensor MRI," J. Magn. Reson. B., 111, 209-219 (1996).

18. F.G. Moeller, K.M. Hasan, J.L. Steinberg, L.A. Kramer, D.M. Dougherty, R.M. Santos, I. Valdes, A.C. Swann1, E.S. Barratt, P.A. Narayana, "Reduced Anterior Corpus Callosum White Matter Integrity is Related to Increased Impulsivity and Reduced Discriminability in Cocaine-Dependent Subjects: Diffusion Tensor Imaging," Neuropsychopharmacology, 30, 610-617 (2005).

19. R.G.M. Schlösser, I. Nenadic, G. Wagner, D. Güllmar, K. von Consbruch, S. Köhler, C.C. Schultz, K. Koch, C. Fitzek, P.M. Matthews, J.R. Reichenbach, H. Sauer, "White matter abnormalities and brain activation in schizophrenia: A combined DTI and fMRI study," Schizophr. Res., 89, 1-11 (2007).

20. D.J. Madden, J. Spaniol, W.L. Whiting, B. Bucur, J.M. Provenzale, R. Cabeza, L.E. White, S.A. Huettel, "Adult age differences in the functional neuroanatomy of visual attention: A combined fMRI and DTI study," Neurobiology of Aging, 28, 459-476 (2007).

21. C. Beaulieu,"The basis of anisotropic water diffusion in the nervous system - a technical review," NMR Biomed, 15, 435-455 (2002).

22. M. Kubicki, C. Westin, R.W. McCariley, M.E. Shenton, "The application of DTI to investigate white matter abnormalities in schizophrenia," Ann. N.Y. Acad. Sci., 1064, 134-148 (2005).

23. A.T. Toosy, O. Ciccarelli, G.J.M. Parker, C.A.M. Wheeler-Kingshott, D.H. Miller, A.J. Thompson, "Characterizing function-structure relationships in the human visual system with functional MRI and diffusion tensor imaging," NeuroImage, 21, 1452-1463 (2004).

24. J.P. Rhinewine, T. Lencz, E.P. Thaden, K.L. Cervellione, K.E. Burdick, I. Henderson, S. Bhaskar, L. Keehlisen, J. Kane, N. Kohn, G.S. Fisch, R.M. Bilder, S. Kumra, "Neurocognitive profile in adolescents with early-onset schizophrenia: clinical correlates," Biol. Psychiatry, 58, 705-712 (2005).

25. R. Nicolson and J.L. Rapoport, "Childhood-onset schizophrenia: rare but worth Studying," Biol Psychiatry, 46, 1418-1428 (1999).

26. T. White, A.T.K. Kendi, S. Lehéricy, M. Kendi, 
C., Karatekin, A. Guimaraes, N. Davenport, S.C. Schulz, K.O. Lim, "Disruption of hippocampal connectivity in children and adolescents with schizophrenia-A voxel-based diffusion tensor imaging study," Schizophr. Res. 90, 302-307 (2007).

27. E.O. Stejskal and J.E. Tanner, "Spin diffusion measurements: spin echoes in the presence of a timedependent field gradient," J. Chem. Phys., 42, 288292 (1965).

28. H. Jiang, P.C. van Zijl, J. Kim, G.D. Pearlson, S. Mori, "DtiStudio: resource program for diffusion tensor computation and fiber bundle tracking," Comput. Methods Programs Biomed., 81, 106-116 (2006).

29. P.J. Basser, J. Mattiello, and D. Le Bihan, "Estimation of the effective self-diffusion tensor from the NMR spin echo," J. Magn. Reson. B, 103, 247-254 (1994).

30. X. Huang, H. Shen, Z. Zhou, X. Wang, W. Hao, D. Hu, "Anisotropic asymmetry analysis in diffusion tensor images of healthy and heroin-dependent subjects," IEEE/ICME International Conference on Complex Medical Engineering, 938-941 (2007).

31. C.D. Good, I.S. Johnsrude, J. Ashburner, R.N.A. Henson, K.J. Friston, R.S.J. Frackowiak, "A voxel-based morphometric study of ageing in 465 normal adult human brains," NeuroImage, 14, 21-36 (2001).

32. J.A. Maldjian, P.J. Laurienti, R.A. Kraft, J.H. Burdette, "An automated method for neuroanatomic and cytoarchitectonic atlas-based interrogation of fMRI data sets," NeuroImage, 19, 1233-1239 (2003).

33. N. Tzourio-Mazoyer, B. Landeau, D. Papathanassiou, F. Crivello, O. Etard, N. Delcroix, B. Mazoyer, M. Joliot, "Automated anatomical labeling of activations in SPM using a macroscopic anatomical parcellation of the MNI MRI single-subject brain," NeuroImage, 15, 273-289 (2002).

34. J.H. Fallon, I.O. Opole, S.G. Potkin, "The neuroanatomy of schizophrenia: circuitry and neurotransmitter systems," Clin. Neurosci. Res., 3, 77-107 (2003).

35. J.N. Giedd, N.O. Jeffries, J. Blumenthal, F.X. Castellanos, A.C. Vaituzis, T. Fernandez, S.D. Hamburger, H. Liu, J. Nelson, J. Bedwell, L. Tran, M. Lenane, R. Nicolson, J.L. Rapoport, "Childhoodonset schizophrenia: progressive brain changes during adolescence," Biol. Psychiatry, 46, 892-898 (1999). 